\title{
$\bullet$ Study on the Efficacy of Silver Nanoparticle Leaf Extract of Pluchea lanceolata
}

\author{
IJCRR \\ Section: Healthcare \\ ISI Impact Factor \\ (2019-20): 1.628 \\ IC Value (2019): 90.81 \\ $\operatorname{SJIF}(2020)=7.893$

\section{Pankaj Nainwal' ${ }^{1}$, Manu Pant ${ }^{2}$}

'Department of Pharmacognosy, School of Pharmacy, Graphic Era Hill University, Dehradun, Uttarakhand, India; 'Assistant Professor,

Department of Life Sciences, Graphic Era Deemed to be University, Dehradun, Uttarakhand, India.

\section{ABSTRACT}

Introduction: A massive contribution is given to the area of current nano-biotechnology by Silver nanoparticles. However a large number of advancements was made to synthesize silver nitrate nanoparticles with different shapes and sizes. Out of the plant extract mediated green procedures are accepted by the research fellows as of their effortless working presence and prepreparations for the protocols.

Objective: Therefore, in the present study use of the leaves extract of Pluchea lanceolata for synthesizing in its silver nanoparticle.

Methods: Silver nitrate nanoparticles were prepared using Fresh leaves of Pluchea lanceolata and characterized by employing X-ray diffraction, spectroscopy and scanning electron microsopy.

Results: The finalized product was characterized using X-ray diffraction (XRD) which shows the Bragg's reflections at $2 \theta$ $=29.7064$, FTIR (Fourier transform infrared spectroscopy) in which the significant peaks were identified at $1602 \mathrm{~cm}^{-1}, 702 \mathrm{~cm}^{-1}$ and $525 \mathrm{~cm}^{-1}$, UV-Visible in which specific signalled peak was identified at $300 \mathrm{~nm}$, Photoluminescence spectroscopy, in which sample was found in an excited state at $300 \mathrm{~nm}$. The morphology was identified with the help of SEM micrographs followed by EDX (Energy Dispersive X-Ray) analysis.

Conclusion: The silver nanoparticles of extract of Pluchea lanceolata were prepared and the efficacy of nanoparticles were studied. The method employed for the formation of silver nanoparticles on the leaves extract of Pluchea lanceolata is costefficient and ecofriendly.

Key Words: $\mathrm{AgNO}_{3}$ nanoparticles, Pluchea lanceolata, Ultra violet, Scanning Electronic Microscopy, X-ray diffraction.

\section{INTRODUCTION}

Nanotechnology is a science deal in controlling substance at the molecular level. Its Incredible growth has opened up various sources of research and development field of science. It is to the lead in various areas of sciences like technology, and various courses of medical sciences. However, the application of metal nanoparticles was also found in drugs and dispensing. Gold $(\mathrm{Au})$, Silver $(\mathrm{Ag})$ nano-particles are mostly employed in making metal nanoparticles in various biomedical uses as well as in nano-biotechnological studies. ${ }^{1}$ There is an excellent application of nanoparticles in the production of nanoparticles in biological as well as in microbiological systems. The application of microorganism in microbiological sciences is increasing because this field is growing success day by day as well as it is very easy to form nano-particles. ${ }^{2}$ Furthermore, the synthesis of metal nanoparticles biologically, is an environment friendly in which use harsh, toxic and expensive chemicals are superseded. Pluchea lanceolata in the indigenous systems of medicine, considered to help treat inflammatory disease and is also indicated in Ayurvedic manuscripts for its therapeutic usefulness in inflammatory disease especially rheumatoid arthritis and other afflictions of joints. In folk uric use the crushed leaves were applied to the inflamed parts of the body. The vaidyas and old herbalist used Pluchea lanceolata drug in the treatment of joint pains or gout. Scientifically, the decoction of the plant has been reported to prevent the swelling of joints. ${ }^{2,3}$ The leaves are found aperients with a variety of applications like a laxative, analgesic, and antipyretic. Quercetin and iso-rhamnetin chemical's presence in the air-dried leaves

\section{Corresponding Author:}

Dr. Pankaj Nainwal, Associate Professor, Department of Pharmacognosy, School of Pharmacy, Graphic Era Hill University, Dehradun, Uttarakhand, India; Phone: 075790296583; Email: drpankajnainwal@gmail.com

ISSN: 2231-2196 (Print)

Received: 10.09 .2020
ISSN: 0975-5241 (Online)

Revised: 13.11 .2020
Accepted: 02.01.2021
Published: 07.05 .2021 
of $P$. lanceolata has been reported so far. Anti-inflammatory potential, antioxidant properties have already been reported in a constituent neolupinol which is isolated from the flowers of this plant. ${ }^{3}$ However, no work has reported before for synthesis of Pluchea lanceolata leaves extract formation with silver oxide nanoparticles, hence an objective was made to perform this study.

\section{MATERIALS AND METHODS}

Analytical grade silver nitrate (AgNO3 $\geq 99.9 \%)$ was purchased without any further purification. Fresh leaves of Pluchea lanceolata was obtained from the Herbal garden, Dehradun, Uttarakhand and was authenticated. The leaves were taken for washing underwater 2-3 times and then again leaves were washed with distilled water. They were then dried in shadow and after drying they have meshed with the help of a grinder then the mashed extract was taken. The extract proceeded for filtration and was filter was used for the synthesis process. $0.1 \mathrm{M} \mathrm{AgNO}_{3}$ solution was prepared and the filtrate was added to it. The mixture was allowed to stand till precipitation occurs. The solution was stirred for 3-4 hours gently. The colour of the solution was gradually changed from colourless to greyish black. The prior formation of silver nanoparticles was estimated by colour change (Figure 1). The solution was again taken for filtration with Whatman's filter paper and the residue was washed 2 times with distilled water. The precipitate was collected in Petri plates. Then it was kept in shelf dryers for drying at 110$120^{\circ} \mathrm{C}$ for 24 hours ${ }^{4}$.

\section{Characterizations}

The detailing and description of structures of the nanoparticles were carried out at room temperature using Analytical XPERT-PRO employing a monochromatic beam of $\mathrm{Cu} \mathrm{K \alpha}$ radiation $\left(1.5407 \mathrm{~A}^{0}\right)$ over $2 \theta$ range of $0^{\circ}-80^{\circ}$. The Fourier Transform I.R. spectrum of the synthesized plant samples was recorded by employing FTIR spectrometer and the UVVisible spectra of the synthesized nanoparticles were recorded in the range $220 \mathrm{~nm}-920 \mathrm{~nm}$ covering the near UV, visible, near-infrared region using CAMAG double beam UV-Vis Spectrophotometer ${ }^{5}$. The fluorometer was then used for recording the photoluminescence range at RT. Scanning electron microscopic analysis was carried out by Scanning electron microscope of GPJ3480 and energy dispersive xrays analysis was done by using highly calibrated Energy Dispersive X-Ray spectrometer.

\section{RESULTS}

\section{X-Ray Diffraction Analysis}

The diffraction pattern of x-rays (Figure 2) demonstrated the Bragg's reflections at $2 \theta=29.7064,32.3086,38.2005$,
46.2865, 54.9546, 57.5901 \& 64.5488, which can be indexed to the (1 11 1), ( $\left.\begin{array}{lll}2 & 1 & 2\end{array}\right),\left(\begin{array}{lll}0 & 1 & 4\end{array}\right),\left(\begin{array}{lll}1 & 1 & 2\end{array}\right),\left(\begin{array}{lll}2 & 14\end{array}\right),\left(\begin{array}{lll}4 & 1 & 3\end{array}\right)$ and (2 2 2) planes of $\mathrm{AgO}$ respectively. These peak heights were found similar to the standard Silver oxide (JCPDS No: 841108). The assessment among all values signifies the presence of silver oxide in every phase which is present in the specimen and was identified as having a tetragonal shape with the constant $\mathrm{a}=6.7902 \mathrm{~A}^{\circ}, \mathrm{c}=9.3659 \mathrm{~A}^{\circ}$. lattices. Figure 3 shows Hall Williamson's plot. The average crystalline material size was found using Scherrer's formula and Hall Williamson plot $^{6}$ as $28.66 \mathrm{~nm}$ and $24.59 \mathrm{~nm}$ respectively.

\section{FTIR Analysis}

This analysis is very much important for the identification of the structure of molecules as well as to seek out the possible numbers of biomolecules present in the leaves extract which was responsible for silver ion reduction and functional group of synthesizing material sample. ${ }^{7}$ However the significant peaks were identified at $1602 \mathrm{~cm}^{-1}, 702 \mathrm{~cm}^{-1}$ and $525 \mathrm{~cm}^{-1}$ which is equivalent to $\mathrm{C}=\mathrm{O}$ stretching, $\mathrm{C}-\mathrm{H}$ bending and vibration occur by silver oxide in series, while besides some other signals or peaks not prominent, were also identified (Figure 4)

\section{UV and Photoluminescence estimation}

For estimation of optical characters, the synthesized crude extract's silver Nanoparticles was evaluated with the help of a UV-Visible (UV-Vis) spectrophotometer at room temperature. In fig 5, a specific signalled peak was identified at $300 \mathrm{~nm}$. The peak was visible with a typical absorbance under a UV spectrophotometer. The visible peak symbolizes the Surface Plasmon Resonance characteristic of silver nanoparticles. The optical band gap of leave extract silver nanoparticles was estimated by employing Tauc's equation which is expressed by $(\alpha \mathrm{h} v)=\mathrm{A}\left(\mathrm{h} v-\mathrm{E}_{\mathrm{g}}\right)^{\mathrm{n}}$. Here $\alpha$ signifies absorption coefficient, A is constant, hv signifies incident light energy, $n$ is exponent whose value relies on the type of transition having values like $1 / 2,2,3 / 2$ and 3 which is similar to allow direct, indirect, forbidden direct, indirect transition respectively. The optical band gap was identified as $3.43 \mathrm{eV}$ from the truck's plot (fig.5). The silver nanoparticle-based extract of Pluchea lanceolata with silver oxide nanoparticles was studied with help of fluoro-emission spectroscopy to know its fluorescence property. ${ }^{8}$ The sample was found in an excited state at $300 \mathrm{~nm}$ and the emission spectra analysis were carried out within a range limit between $200-900 \mathrm{~nm}$, based on excitation maxima (Fig 6). The peak at $380 \mathrm{~nm}$ (eg $=3.3 \mathrm{eV}$ ) was nearly coinciding with the UV Spectra. The peaks at $520 \mathrm{~nm}$ and $790 \mathrm{~nm}$ were because of the occurrence of oxygen vacancy and surface imperfections respectively.

\section{SEM with EDX Analysis}

To study the structures of nanoparticles, a typical analysis from SEM (Scanning Electron Microscope) was used. The 
signals of elemental metal detection were observed by the Energy Dispersive X-Ray method for confirmation. The surface morphological detection of the silver oxide Nanoparticles was done at different magnifications ranging from 10000 to 55000 (Fig.7). The images obtained from the scanning electron microscope depicts that the density of silver nano-particles are high and also gave confirmation regarding the development of silver nanostructures. ${ }^{9}$ The aggregation of particles are observed however the surfaces between single crystals were identified and observed. The result of the Energy Dispersive X-Ray shows that there is a higher count at 3 kiloelectron volts because of the presence of silver nanoparticles (fig 8) and this is revealed by representing a typical optical absorption peak at $3 \mathrm{keV}$. It might be due to surface plasmon resonance, which also represents the occurrence of oxygen. The particles have seen rough spherical shapes.

\section{DISCUSSION}

In the present study, the method employed for the formation of silver nanoparticles on the leaves extract of Pluchea lanceolata is cost-efficient and ecofriendly. The X-ray diffraction methods were employed to identify the size and morphology of prepared extract silver nanoparticles. The green method in form of usage of eco-friendly reducing agent used in FTIR spectroscopic analysis shows the occurrence of the functional group similar to phytoconstituents present in plants. ${ }^{6,7,8}$ These phytochemical acts both capping and reductant in the synthesis process. The Silver oxide vibration by using FTIR in test samples of plant extracts showed functional groups. The optical band gap identified to be 3.42 and 3.31 from Ultraviolet and Photo luminance analysis respectively. The contrast that occurs in between was due to the stokes shift. The scanning electron microscopic analysis results from the roughly spherical structure of the nanocrystallites. Elemental compositions were analyzed by the EDAX spectrum. ${ }^{9}$

\section{CONCLUSION}

This study, also results out that if there is an increase in the concentration of silver nitrate in solution then there will be an increase in the density of nanoparticles which enhances the growth of large particles from nanoparticles. As some evidence results during the UV-Vis spectra in which there is an increase in absorbance and redshift of SPR peaks, which proves the above-said matter. The solution's retention time also affect the silver nanoparticle's stability and size, as particle size increase with the increase in retention time which was proved by the formation of a secondary peak in UV-Vis spectra.

\section{ACKNOWLEDGEMENT}

I would like to thanks Chancellor, Vice-Chancellor, Director Research and Director Pharmacy to provide all amenities and funds for this project.

Source of Fund: This project was approved for funding under Faculty Development Programme 2019, by Graphic Era Hill University, Dehradun, Uttarakhand.

\section{Conflict of Interest: None}

\section{REFERENCES}

1. Siavash I. Green synthesis of metal nanoparticles using plants. Green Chem 2011;13:2638-2650.

2. Prasob Peter KJ. Multi-functional silver nanoparticles for drug delivery: A review. Int J Cur Res Re. 2017;9(8):1-5.

3. Gupta RK. Medicinal and aromatic plants. CBS publishers and distributors. Edition 1st. 2010: 468-469.

4. Srivastava P, Shanker K. Pluchea lanceolata (Rasana): Chemical and biological potential of Rasayana herb used in traditional system of medicine. Fitoterapia 2012;83(8):1371-1385.

5. Sundarrajan M, Jeelani A, Santham V, Durgadevi S, Abirami S. Effect of concentration, ph and time on the morphology of silver nanoparticles synthesized by the green method. Int J Cur Res Rev 2018;10(21);25-29.

6. Irfana AM, Jothi MR, Mubeen F. Green Synthesis of Copper Oxide Nano Particles Using Moringa Oleifera Leaf Extract. Int J Trend Dev 2018;15:17-18.

7. Subramaniyam R, Veeranna P, Kumar G. A novel approach for the biosynthesis of silver oxide nanoparticles using aqueous leaf extract of Callistemon lanceolatus (Myrtaceae) and their therapeutic potential. J Exp Nanosci 2016;11(6):445-458.

8. Saxena A, Tripathi RM, Zafar F, Singh P. Green synthesis of silver nanoparticles using aqueous solution of Ficus benghalensis leaf extract and characterization of their antibacterial activity. Materials Let 2012;67:91-94.

9. Varthini T, Carmel Vijila GM, Jothi MR. Effect of Medicinal Leaf Extract In Silver Nitrate -Size Reduction To Nanoscale. J Emerg Tech Innov Res 2018;5(6):664-666. 


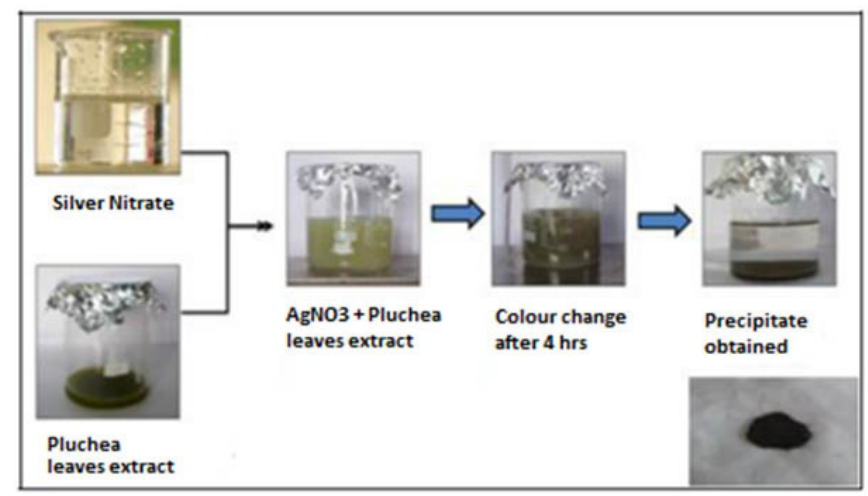

Figure 1: Reaction process using P.lanceolata and obtained AgO particles

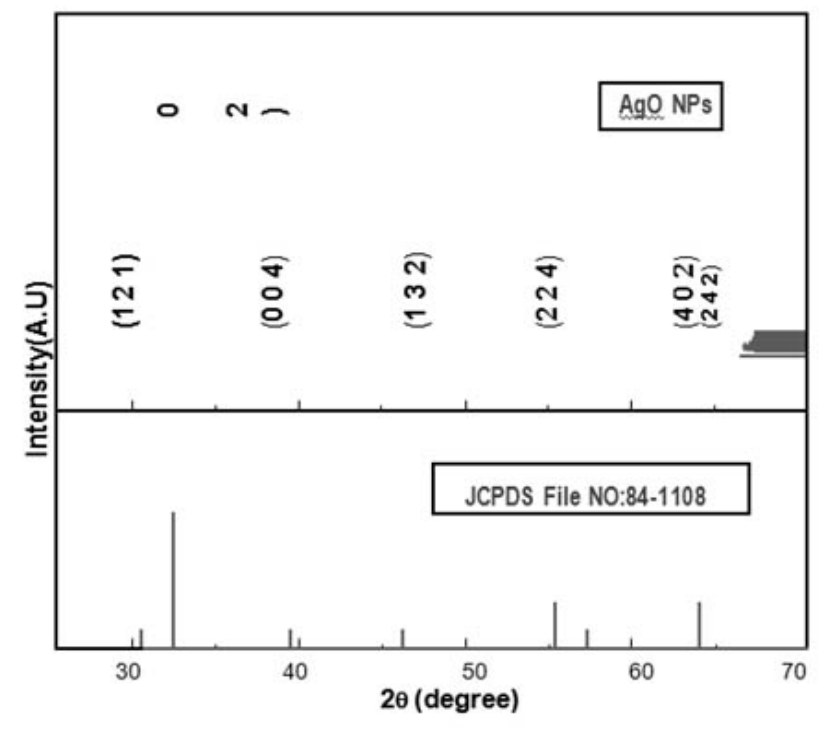

Figure 2: X- ray Diffraction pattern.

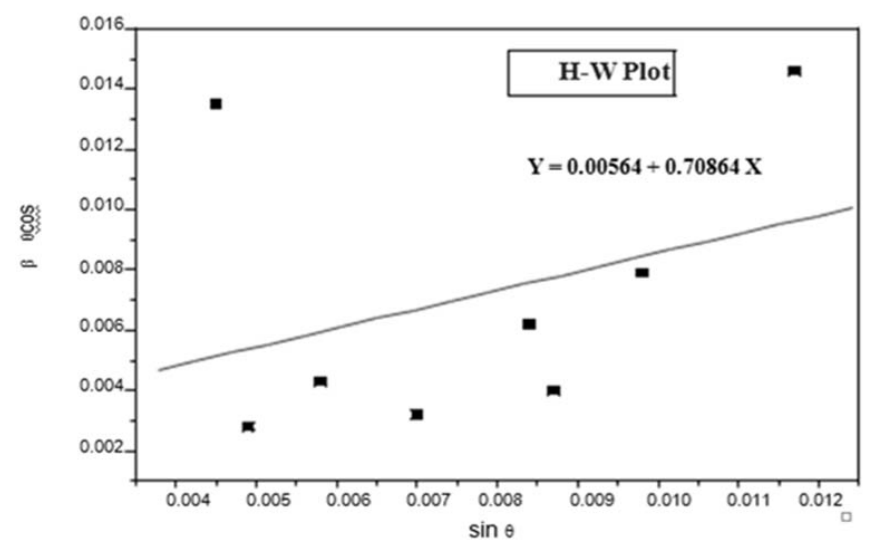

Figure 3: Hall Williamson plot of AgO Nanoparticles

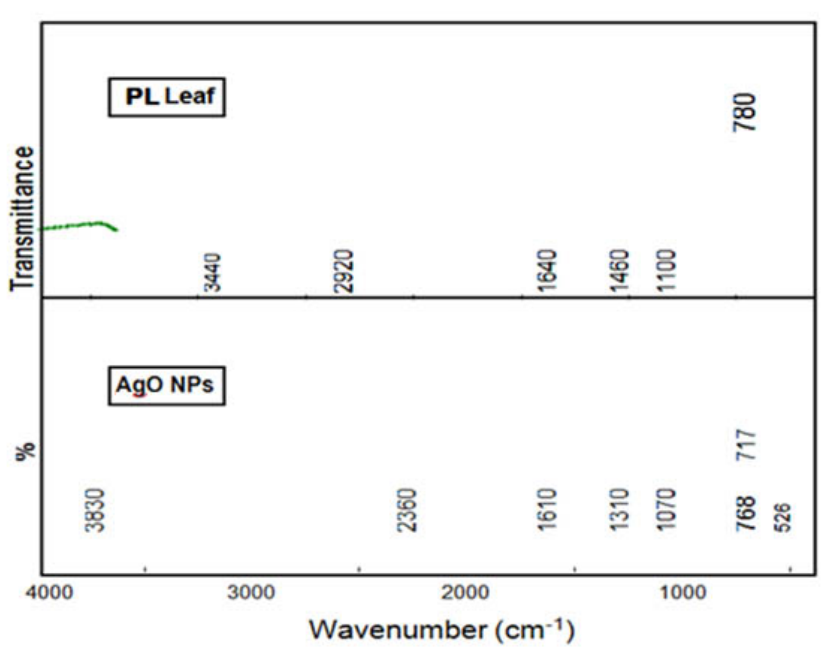

Figure 4: FTIR Spectra of Pluchea lanceolata leaves and AgO nanoparticles

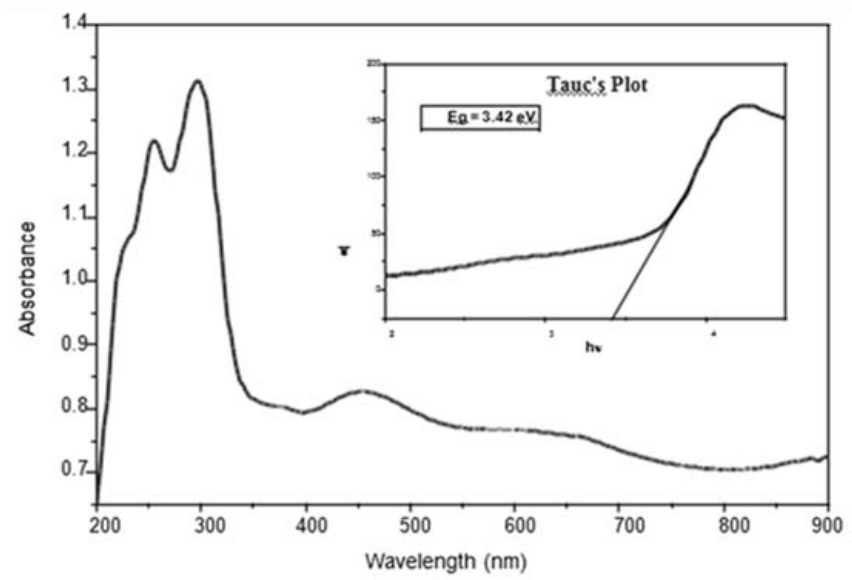

Figure 5: UV Absorbance Spectrum and Tauc's Plot

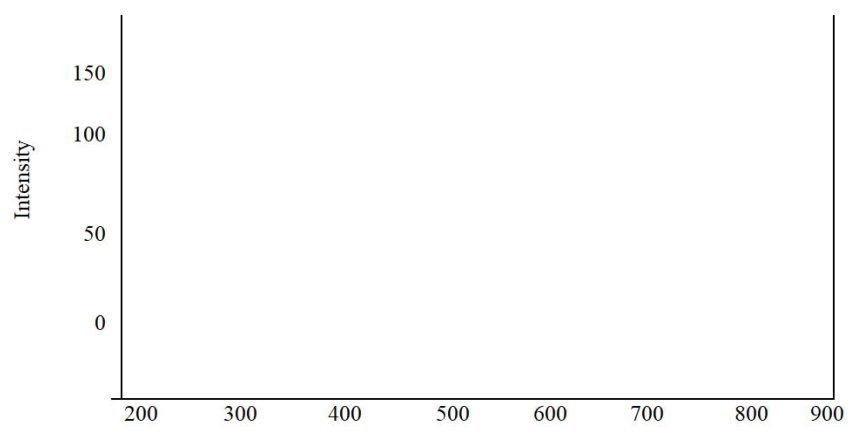

Figure 6: Photoluminescence Spectrum 


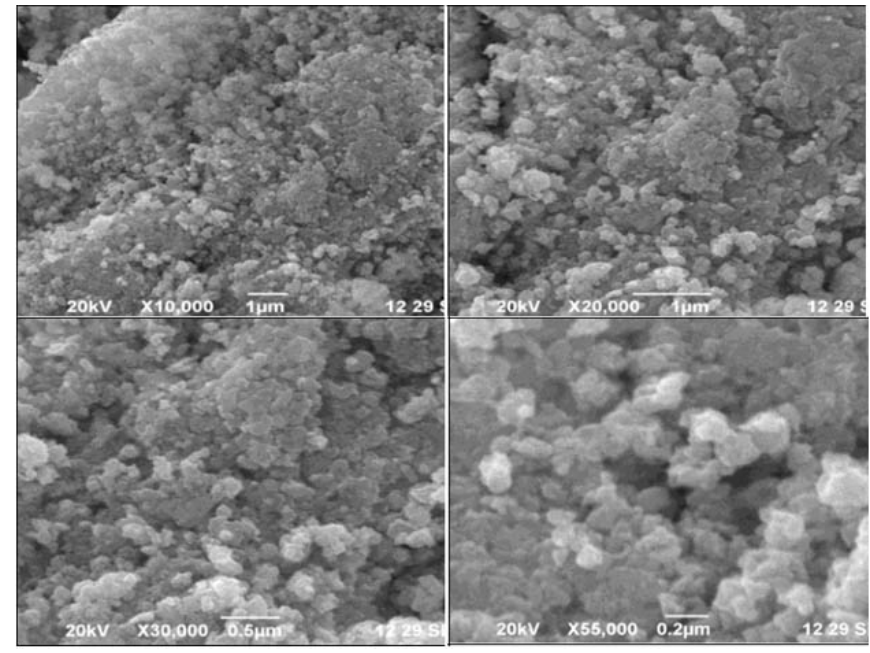

Figure 7: SEM micrographs of AGSO at different magnifications (a) $\times 10,000$ (b) $\times 20,000$ (c) $\times 30,000$ (d) $\times 55,000$

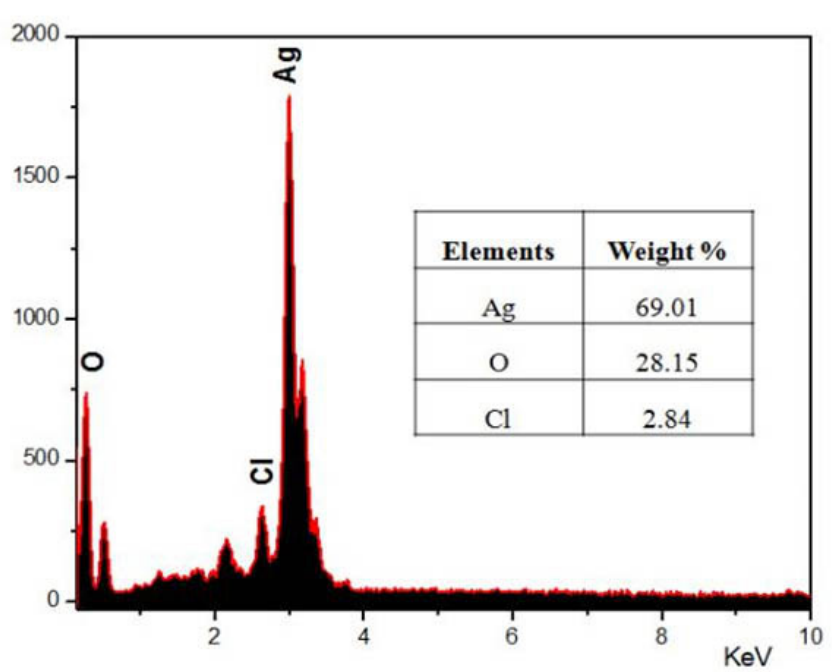

Figure 8: EDX Spectrum of Ago Nanoparticles. 